csDMARDs were introduced: methotrexate (MTX), leflunomide (LFN), sulfasalazine (SSZ) and hydroxychloroquine (HCQ). In addition, the following bDMARDs were used: abatacept, adalimumab (ADA), certolizumab, etarnecept (ETN), golimumab, infliximab (INF), rituximab and tocilizumab. Finally, small doses of steroids were used. During follow-up the clinical, laboratory findings as well as the treatment decisions and strategies were all recorded. In addition, the adverse drug reactions, the reason of termination or changing strategies, disease complications and comorbidities were all recorded. Finally, disease activity was measured with the 28 joint count, Disease Activity score-28 (DAS-28) using the erythrocyte sedimentation rate.

Results: All patients have received one csDMARD, at least for 6 months. The csDMARD of first choice was MTX (58\%), followed by LFN (32\%), HCQ (8\%), and SSZ $(2 \%)$ with or without small doses of steroids. The bDMARD of first choice was INF (37\%) followed by ETN (32\%) and ADA (31\%). During the follow up period, 14 patients were lost. In addition, 7 patients never received bDMARDs due to various comorbidities. Thus, the final results are referred to 517 patients. Among those patients, $324(66 \%)$ were treated with csDMARDs as monotherapy or in combination therapy with or without the use of steroids with significant clinical improvement and sustained LDA. However, eleven patients (3,2\%) from this group neither achieved LDA, nor received bDMARDs, due to comorbidities. On the other hand, 175 patients $(34 \%)$ were treated with bDMARDs with or without csDMARDs or/ and steroids. The majority of them demonstrated sustained LDA for a long period of time. From this group 31 patients $(17,7 \%)$ never achieved LDA, despite that they switched and received all bDMARDs. Thus a total of $20,9 \%$ of our patients never achieved LDA.

Conclusions: Using the EULAR and ACR recommendations for RA therapy we successfully treated the majority of our patients. However, we found that the size of gap and the unmet needs for RA treatment is about twenty per cent. This is the first study aiming to estimate the gap and the size of unmet needs for RA treatment in a large patient population followed-up in a tertiary university centre.

Disclosure of Interest: None declared

DOI: 10.1136/annrheumdis-2018-eular.3392

\section{FRI0102 REDUCTION OF ANTIDRUG ANTIBODY LEVELS AFTER SWITCHING TO RITUXIMAB IN PATIENTS WITH RHEUMATOID ARTHRITIS WITH PREVIOUS FAILURE TO INFLIXIMAB OR ADALIMUMAB}

A. Martinez-Feito ${ }^{1}$, C. Plasencia ${ }^{1}$, V. Navarro-Compan ${ }^{1}$, B. Hernández-Breijo ${ }^{1}$, D. Pascual-Salcedo ${ }^{1}$, P. Nozal ${ }^{2}$, C. Diego ${ }^{2}$, I. Monjo ${ }^{1}$, L. Nuño ${ }^{1}$, A. Balsa ${ }^{1}$

${ }^{1}$ Immuno-Rheumatology research group; ${ }^{2}$ Immunology, University Hospital La Paz, Madrid, Spain

Background: Rituximab (Rtx), a monoclonal antibody against CD20+, induces transient depletion of $B$ cells and was approved for the treatment of patients with active rheumatoid arthritis (RA). Previous data ${ }^{1}$ showed that Rtx is particularly effective on autoimmune diseases in which auto-antibodies (auto-Ab) are produced. Based on this, it is hypothesised that auto-Ab produced by short-lived plasma cells expressing CD20 are targets of Rtx. Given this scenario, the immunogenicity related to the use of Infliximab (Ifx) or Adalimumab (Ada) could be cleared by Rtx.

Objectives: First, to analyse the persistence of anti-drug antibodies (ADA) after switching to either a 2nd biological therapy (TNFi or Rtx) after 12 months of followup. Second, to evaluate whether the reduction of ADA levels are influenced by the mechanism of action of the second biological therapy (BT).

Methods: Dataset from a prospective cohort including all patients with RA starting BT at the Unit of Complex Therapy in a tertiary hospital was used. For this study, data from 21 patients who had experienced previous failure to Ifx (57\%) or Ada (43\%) related to ADA detection and then switched to a 2nd TNFi (Ifx, Ada, Etanercept or Certolizumab) or to Rtx was analysed. Additionally, patients should have both determinations of ADA levels: at the end of the first BT and 12 months after switching. ADA were determined by a bridging ELISA. The proportion of patients with ADA positive levels at 12 months was determined. Also, the relative reduction (in median) of ADA levels between patients switching to a 2nd TNFi vs Rtx in both visits was compared

Results: Out of 21 ADA positive patients with RA, 15 (71\%) switched to a 2nd TNFi and $6(29 \%)$ to Rtx. Demographic characteristics of patients are shown in table 1.

ADA remained positive in $80 \%$ of patients at 12 months after switching $B T$. The percentage of patients with undetectable ADA levels at 12 months was higher in the group of patients treated with Rtx compared with patients receiving a 2nd TNFi ( $33 \%$ vs $13 \%$, respectively). The median ADA levels $(\mathrm{AU} / \mathrm{ml}$ ) values at baseline and at 12 months were 536 and 123 for Rtx and 3625 and 1842 for TNFi. The relative reduction of median ADA levels between baseline and 12 months visits was higher in patients with Rtx than in patients treated with $\mathrm{TNFi}(-78 \%$ vs $-50 \%$, respectively).

Abstract FRI0102 - Table 1. Demographics characteristics of the 21 patients according to the second biologic.

\begin{tabular}{lcc}
\hline Switch to: & TNFi(n=15) & RTX(n=6) \\
\hline Women, $\mathrm{n}(\%)$ & $13(87 \%)$ & $6(100 \%)$ \\
Age, years, median(IQR) & $47\left({ }^{37-56}\right.$ & $50.5(42.5-65.5)$ \\
Smoking status, $\mathrm{n}(\%)$ & $2(13 \%)$ & $1(17 \%)$ \\
$\mathrm{IMC}, \mathrm{Kg} / \mathrm{m}^{2}$, median(IQR) & $23.6(19.3-28.6)$ & $34.1(25.2-32.5)$ \\
\hline
\end{tabular}

Conclusions: Despite discontinuing TNFi, ADA titters remain positive in a high proportion of patients with RA after one year. Over time, ADA levels decrease in patients who switch to a second $\mathrm{BT}$, being this effect more pronounced in patients receiving $\mathrm{Rtx}$ than a 2 nd $\mathrm{TNFi}$. This effect could be explained by the intrinsic action mechanism of Rtx on plasmatic CD20 +cells.

\section{REFERENCE:}

[1] Huang $\mathrm{H}$, et al. Rituximab specifically depletes short-lived autoreactive plasma cells in a mouse model of inflammatory arthritis. PNAS2010 Mar 9;107(10):4658-63.

Disclosure of Interest: None declared

DOI: 10.1136/annrheumdis-2018-eular.5545

\section{FRI0103 ONE-YEAR FOLLOW-UP OF A NATIONWIDE COHORT OF PATIENTS WITH INFLAMMATORY ARTHRITIS, WHO SWITCHED FROM ORIGINATOR TO BIOSIMILAR ETANERCEPT, FOCUSING ON PATIENTS WHO SWITCHED BACK TO ORIGINATOR. AN OBSERVATIONAL DANBIO STUDY}

B. Glintborg, I.J. Sørensen, E. Omerovic, F. Mehnert, N. Manilo, K. Danebod, D. V. Jensen, H. Nordin, A.G. Loft, O. Hendricks, S. Chrysidis, B.L. Andersen, J. L. Raun, H. Lindegaard, J. Espesen, S.H. Jakobsen, I.M.J. Hansen, E.

B. Dalsgaard, D.D. Pedersen, S. Kristensen, A. Linauskas, L.S. Andersen, J. Grydehøj, N.S. Krogh, M.L. Hetland. The DANBIO registry and the Danish Departments of Rheumatology, Copenhagen, Denmark

Background: In Denmark, patients (pts) treated with originator etanercept (ETA) $50 \mathrm{mg}$ SC conducted a mandatory non-medical switch to biosimilar SB4 in April 2016 (switchers). Pts treated with $25 \mathrm{mg}$ ETA or $50 \mathrm{mg}$ powder-solution were not mandated to switch (non-switchers). Some switchers resumed ETA during followup (back-switchers)

Objectives: To investigate the frequency of back-switching after the non-medical switch from ETA to SB4, and in back-switchers to study, 1) baseline characteris tics at the time of initial switch (ETA->SB4), 2) reasons for SB4 withdrawal, 3) changes in disease activity during treatment with SB4 and after back-switching.

Methods: Patient data were retrieved from the DANBIO registry (censored August 2017). For back-switchers, disease activity at the start of SB4 (=baseline) and at the time of back-switching to ETA were compared, and changes in disease activity between the two time points were calculated (=delta values), stratified by indication (RA/PsA/AxSpA). Baseline characteristics of back-switchers were compared to the rest of the switch population (Chi-sq, Mann-Whitney U-test). Abbreviations are shown in table 1.

Results: 1641 pts switched from ETA to SB4. Of these, 299 (18\%) withdrew SB4 therapy during 1 year follow-up and either switched back to ETA $(n=120,7 \%)$ started another bDMARD $(n=104)$, died $(n=9)$, were lost to follow-up $(n=1)$ or did not re-start bDMARDs $(\mathrm{n}=65)$

Among the 120 back-switchers, SB4 was withdrawn due to LOE (52\%), AE $(39 \%)$, or other/unknown reasons $(9 \%)$. The reasons for withdrawal of SB4 in back-switchers are listed in table 1. No major safety events occurred. The median time on SB4 before back-switching was 120 (IQR 73-193) days, and the time 
